\title{
Online tuning of impedance matching circuit for long pulse inductively coupled plasma source operation - an alternate approach
}

\author{
Dass Sudhir $^{1}$, M. Bandyopadhyay ${ }^{1}$, W. Kraus ${ }^{3}$, A. Gahlaut ${ }^{2}$, G. Bansal $^{2}$ and A. Chakraborty ${ }^{1}$ \\ ${ }^{1}$ ITER-India, Institute for Plasma Research, A-29, GIDC, Sector-25,, Gandhinagar, Gujarat, 382 025, India. \\ ${ }^{2}$ Institute for Plasma Research, Bhat, Gandhinagar, Gujarat, 382 428, India. \\ ${ }^{3}$ Max-Planck-Institut für Plasmaphysik, Boltzmannstrasse 2, 85740 Garching, Germany.
}

\begin{abstract}
Impedance matching circuit between RF generator and the plasma load, placed between them determines the RF power transfer from RF generator to the plasma load. The impedance of plasma load depends on the plasma parameters through skin depth and plasma conductivity or resistivity. Therefore, for long pulse operation of ICPs, particularly for high power ( $100 \mathrm{~kW}$ or more) where plasma load condition may vary due to different reasons (e.g. pressure, power, thermal etc.), online tuning of impedance matching circuit is necessary through feedback. In fusion grade ion source operation such online methodology through feedback is not present but offline remote tuning by adjusting the matching circuit capacitors and tuning the driving frequency of the RF generator between the ion source operation pulses is envisaged. The present model is an approach for remote impedance tuning methodology for long pulse operation and corresponding online impedance matching algorithm based on RF coil antenna current measurement or coil antenna calorimetric measurement may be useful in this regard.
\end{abstract}

\section{Introduction:}

Radio frequency driven (RF) inductively coupled plasma (ICP) discharges have been known for many years $[1,2]$. They provide very efficient, economical and technologically simple way of producing high density uniform plasmas in field of semiconductor $[3,4]$ and fusion technology $[5,6]$. In fusion grade machines, neutral beam injector (NBI) systems and its related R\&D facilities in Europe [7] and in India [8], ICP based negative ion sources are planned to be used due to its low maintenance requirements $[9,10]$. Geometrical parameters like length, diameter and number of turns of the coil antenna play a big role in coupling RF power into the plasma in ICP [11]. The RF power transfer from RF generator to the plasma depends on the impedance matching circuit placed between RF generator and the plasma load. The impedance of plasma load depends on the plasma parameters through skin depth and plasma conductivity. Plasma load condition may vary due to different reasons (e.g. pressure, power, thermal etc.). As a result online impedance matching is desirable through feedback loop. In low power ICPs, it is normally done with measuring reflected power from the load using directional coupler in a feedback mechanism. In fusion grade ion source operation [7, 8], such online methodology is not present but remote tuning by adjusting the driving frequency of the RF generator between the ion source operation pulses is envisaged 
A set of analytical formulation is presented based on air core transformer model [12, 13, 14]. In the model, RF antenna coil is considered as transformer primary coil and the plasma as the single turn secondary coil. The variable plasma characteristics are folded into the matching algorithm which enable to characterize plasma from external electrical parameters. The model can be used to calculate RF power coupling to the plasma in terms of coil antenna cooling water calorimetric data and measurable electrical circuit parameters which can be linked to the matching circuit RF generator's variables like capacitance and driving frequency. Single RF driver model calculations are validated with experimental data, obtained from $\operatorname{ROBIN}[15,16]$, which is a single driver negative ion source driven by $1 \mathrm{MHz}$ RF generator.

The present model is an approach for online remote impedance tuning methodology based on RF coil antenna current measurement or coil antenna calorimetric measurement. An algorithm is also described based on this methodology which can be implemented through a feedback control program into a motor based driving system to tune the variable capacitors mechanically either in the matching circuit or in the tank circuit inside the RF generator unit.

\section{Description of the ICP setup:}

Single Driver based: The plasma in the ICP is created inside an RF driver consists of 6.5 turn water cooled copper coil wrapped on an alumina cylinder. The copper coil is a tube with $6 \mathrm{~mm}$ OD and $4 \mathrm{~mm}$ ID. The RF coil on the source is connected to the $1 \mathrm{MHz}, 100 \mathrm{~kW}$ RF generator through an impedance matching network, as shown in Figure 1. The RFG is based on selfexcited tetrode oscillator configuration with capacitive feedback [17]. The matching circuit is a combination of the series (C2) and shunt (C1) capacitances and a 3:1 transformer in the shunt arm. The transformer helps to isolate the source from the generator in the event of source being floated at high potential during beam extraction and acceleration. The detailed description is given in refs. [15, 16].

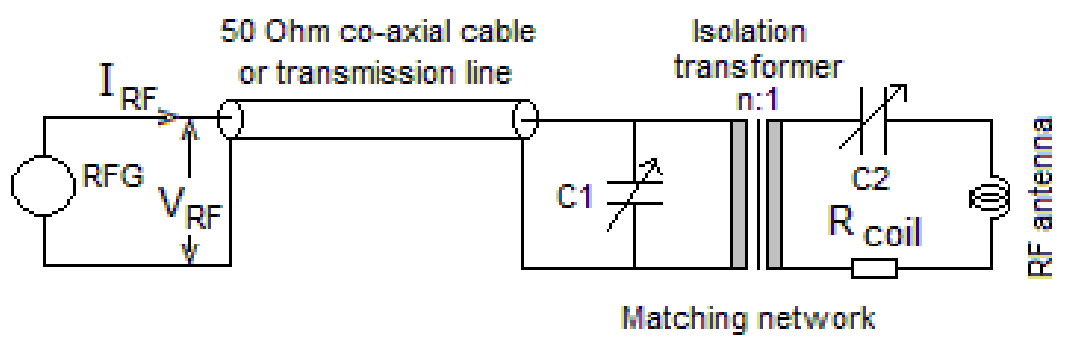

Figure 1: Schematic of the matching circuit connected to the RF antenna coil. RF generator (RFG) having output voltage $V_{R F}$ and current $I_{R F}$ is connected to the RF antenna through $50 \Omega$ co-axial transmission line and matching circuit, comprising of C1, C2 variable capacitors and an insolation transformer of turns ratio n:1. Antenna coil circuit resistance is denoted by $R_{\text {coil }}$. 


\section{Description of the model:}

\section{(a) Air core transformer model for ICP for calculation of plasma load:}

Plasma in a RF discharge can be considered as an electrically conductive fluid, comprising of multitude of filamentary discharges that essentially run in parallel. Therefore, the plasma in an inductive discharge forms a one turn secondary winding of an inductance $L_{m p}$, with respect to the driver coil as primary of an air-core transformer. The corresponding resistance of the plasma is $R_{p}$. The transformer model is depicted in fig.(2a and 2b). In the fig.(2c), the secondary circuit $L_{s}$ is the equivalent discharge inductance which consists of two components, i) plasma inductance $L_{p}$ due to electron inertia which follows the plasma conductivity formula and ii) the geometric (or magnetic) inductance $L_{m p}$ which is due to the discharge current path. $L_{m p}$ is inductively coupled to the primary coil through mutual inductance $M$. These figures do not include the electrical parameters of the matching circuit and only depict the coupling phenomena between the coil antenna and the plasma, based on transformer model [14].

(a)

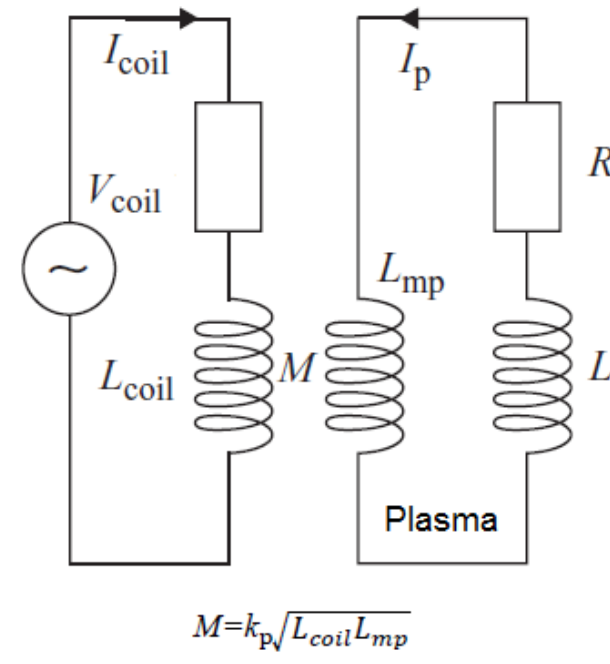

(b)

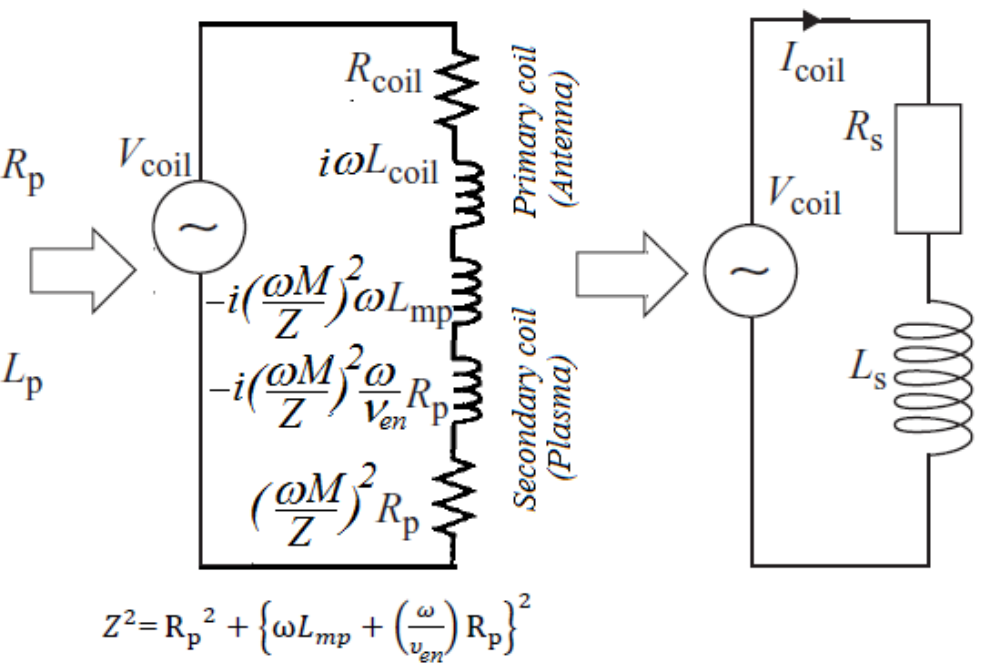

Figure 2. (a) Depiction of transformer model of the RF driver [14]. Left side figure is the antenna coil (primary side of the transformer) physically connected to the RF Generator through matching circuit. $L_{\text {coil }}$ is the antenna coil inductance; $V_{\text {coil }}$ and $I_{\text {coil }}$ are the voltage and current respectively applied across the coil due to RF Generator. Right hand side figure is the plasma (single turn secondary, inductively coupled with primary coil). $M$ is the mutual inductance between the coil and plasma, $L_{m p}$ is inductance, $R_{p}$ is the resistance of the secondary (plasma) and $I_{p}$ is the plasma current passing in the secondary circuit. (b) Equivalent circuit of the transformer model, identifying values of individual components and its contribution in terms of measurable quantities. (c) Simplified equivalent circuit of the transformer model. $L_{s}$ 
and $R_{s}$ are the effective inductance and resistance seen by the RF Generator, used for tuning calculation.

Through a straightforward circuit analysis the coupled circuits can be transformed into simpler form as shown in fig 2 . In this representation the secondary circuit elements are written in terms of the primary circuit current. Applying Kirchhoff's law in above circuit we get [17],

$$
\begin{gathered}
V_{\text {coil }}=i \omega L_{\text {coil }} I_{\text {coil }}+R_{\text {coil }} I_{\text {coil }}+i \omega M I_{p} \\
V_{p}=i \omega L_{m p} I_{p}+i \omega M I_{\text {coil }}=-I_{p}\left[R_{p}+i R_{p}\left(\frac{\omega}{v_{e n}}\right)\right] \\
V_{\text {coil }}=\left(i \omega L_{s}+R_{S}\right) I_{\text {coil }}
\end{gathered}
$$

Here $\omega$ is the driver frequency, $M$ is the coefficient of mutual inductance between the coil and the plasma, $R_{p}$ is the plasma resistance, $L_{m p}$ is the plasma inductance due to plasma current discharge loop, $L_{e p}=\frac{R_{p}}{v_{e n}}$ is the inductance due to electron inertia effect where $v_{e n}$ is the electron neutral collision frequency. In the circuit, $I_{p}$ is induced plasma current and $V_{p}$ is induced plasma voltage, $V_{\text {coil }}$ is the voltage across the driver.

After incorporating air core transfer action between antenna and plasma with plasma impedance, and solving above set of equations, effective inductance and resistance across coil during coupling can be given as,

$$
\begin{aligned}
& L_{s}=\left\{L_{\text {coil }}-M^{2} \omega^{2}\left[\frac{\left(L_{m p}+\frac{R_{p}}{v_{e n}}\right)}{R_{p}^{2}+\omega^{2}\left\{L_{m p}+\left(\frac{R_{p}}{v_{e n}}\right)\right\}^{2}}\right]\right\}, \\
& R_{S}=\left\{R_{\text {coil }}+M^{2} \omega^{2}\left[\frac{R_{p}}{R_{p}^{2}+\omega^{2}\left\{L_{m p}+\left(\frac{R_{p}}{v_{e n}}\right)\right\}^{2}}\right]\right\}, \\
& M^{2}=k_{p}^{2} L_{\text {coil }}\left(L_{m p}+\frac{R_{p}}{v_{e n}}\right) \approx k_{p}^{2} L_{\text {coil }} L_{m p},
\end{aligned}
$$

where $R_{\text {coil }}$ and $L_{\text {coil }}$ are two measured quantities and voltage appeared across RF driver coil antenna is $V_{\text {coil }}$ which is $1 / n$ times of $V_{R F}$, output voltage of RF generator, where $\mathrm{n}$ is the transformer ratio of the isolation transformer (shown in fig. 1). The $2^{\text {nd }}$ term inside the curly brackets in equation (4) and (5) are induced terms and $R_{p}^{2}+\omega^{2}\left\{L_{m p}+\left(\frac{R_{p}}{v_{e n}}\right)\right\}^{2}$ is the total impedance of the plasma, However, $\left(\frac{R_{p}}{v_{e n}}\right)$ term is extremely small and can be neglected in the impedance part in equation (6). The negative sign in inductance associated with the secondary reactance (eq-4) can be simply understood by noting that current in the secondary causes some of 
the magnetic flux in the primary to be neutralized, thus resulting in a lower total magnetic flux in the primary circuit.

The equations (4), (5) are depicted in fig.2 (b). Based on fig.2 (c), $R_{s}$ and $L_{s}$ can be quantified from measurable parameters $V_{\text {coil }}, I_{\text {coil }}, \omega$ and $\phi$ from RF generator in the following way using power relations [14].

$$
\begin{aligned}
R_{S} & =\frac{V_{\text {coil }}}{I_{\text {coil }}} \cos \phi \\
L_{S} & =\frac{V_{\text {coil }}}{\omega I_{\text {coil }}} \sin \phi .
\end{aligned}
$$

In the above equations (7) and (8), $\phi$ is the phase angle between RF current and voltage applied to the coil. $I_{\text {coil }}$ can be measured directly or estimated by calorimetric data from the coil cooling circuit considering joules heating of the coil due to the passing of the current $I_{\text {coil }}$ through the coil antenna.

Direct measurement of coil current $I_{\text {coil }}$ will be more accurate. However sometimes current measuring devices are not allowed in the system due to some maintenance, electromagnetic noise or radioactive related issues. In such cases, calorimetric approach as an alternate technique to calculate the coil current can be adopted. To estimate the error in calorimetric approach a calibration may be necessary and corresponding calibration factor can be incorporated in the calculation to adjust the error.

A third equation (needed for an analytical solution) follows directly from the basic premise of this work that the plasma is a one-turn current path that surrounds the primary coil. As it is known that, inductance of any coil of radius $r_{\text {coil }}$ and number of turns $n_{\text {coil }}$ can be represented as, $L_{\text {coil }} \propto\left(n_{\text {coil }} \cdot r_{\text {coil }}\right)^{2}$. In the same light inductance of single turn plasma is $L_{m p} \propto r_{\text {plasma }}{ }^{2}$. Hence it can be written as,

$$
L_{m p}=L_{\text {coil }}\left(\frac{k_{p}}{n_{\text {coil }}^{2}}\right)
$$

For the present case (coil is outside the plasma) coupling parameter $k_{p}$ can safely be taken as $k_{p} \approx\left(\frac{r_{\text {plasma }}{ }^{2}}{r_{\text {coil }^{2}}}\right)[14]$.

The plasma dependent quantities $R_{p}, L_{m p}$, are needed that can be found from solving these above two equations of $L_{s}$ and $R_{s}$, equations (6) and (7) with equations (9).

$$
L_{m p}=L_{\text {coil }} \frac{\left(\sqrt{\frac{\left[\omega^{2}\left(L_{\text {coil }}-L_{S}\right)^{2}+\left(R_{S}-R_{\text {coil }}\right)^{2}\right]}{L_{\text {coil }} \omega^{2}\left[\left(L_{\text {coil } \left.\left.-L_{S}\right)-\frac{1}{v_{e n}}\left(R_{S}-R_{\text {coil }}\right)\right]}\right.\right.}}\right)}{n_{\text {coil }}{ }^{2}},
$$




$$
R_{p}=L_{\text {coil }}\left(\frac{1}{n_{\text {coil }}^{2}}\right)\left(\sqrt{\frac{\left[\omega^{2}\left(L_{\text {coil }}-L_{S}\right)^{2}+\left(R_{S}-R_{\text {coil }}\right)^{2}\right]}{L_{\text {coil }} \omega^{2}\left[\left(L_{\text {coil }}-L_{S}\right)-\frac{1}{v_{\text {en }}}\left(R_{S}-R_{\text {coil }}\right)\right]}}\right)\left(\frac{1}{\frac{1}{v_{e n}}-\left[\frac{L_{\text {coil }}-L_{S}}{\left.R_{S}-R_{\text {coil }}\right]}\right.}\right) .
$$

The values of plasma resistance $R_{p}$ and impedance $L_{m p}$ in equations (10) and (11) are calculated using measurable quantities obtained from electrical circuits connected to the RF Generator. The measurable quantities are, $n_{\text {coil }}, L_{\text {coil }}, V_{\text {coil }}, I_{\text {coil }}, \cos \phi, \omega$, and the pressure (for collision frequency $\left.v_{e n}\right)$.

Measureable values of $V_{\text {coil }}, I_{\text {coil }}, \cos \phi, \omega$ are used to estimate $L s$ and $R s$ from equations (7) and (8); $L_{\text {coil }}, \omega, v_{e n}$ (from pressure value) and also using the above set of equations $(10-11), R_{p}, L_{m p}$ which are functions of plasma parameters can be computed. The values $R_{p}$ and $L_{m p}$ can be used in another model for RF impedance matching circuit analysis, which is discussed in the following section. It is observed that the inductance of the plasma secondary, $L_{m p}$ is small compared to primary coil inductance $L_{\text {coil }}$.

The present model is an electrical model where physics pictures are not included adequately. Incorporation of different physics phenomena including non-linear effects in high power ICPs are being carried out and will be presented elsewhere.

\section{(b) Model to include plasma impedance variation into RF matching:}

Plasma impedance depends on plasma parameters. The equivalent matching circuit including plasma as a load is shown in fig3 . In this figure $L_{m p}$ is not considered due to its extremely small values.

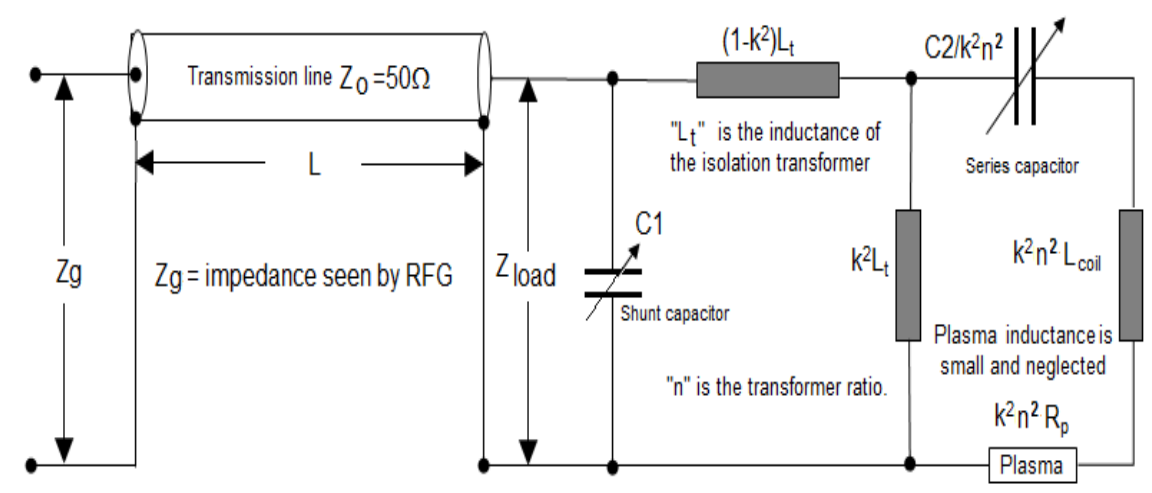

Figure 3. Impedance matching electrical equivalent circuit diagram. $Z_{\text {load }}$ is the net load at transmission line end due to the antenna coil impedance, plasma resistance $R_{p}$ and that of matching circuit - comprising of isolation transformer of inductance $L_{t}$ and two variable capacitors $C 1, C 2$. Net impedance seen by the $R F$ generator is $Z_{g}$ which includes $Z_{\text {load }}$ and the transmission line impedance $Z_{0}=50 \Omega$. The inductance of the isolation transformer is $L_{t}$ where, $k$ is the coupling factor between primary and 
secondary turn of it. Except plasma resistance $R_{p}$, other parameters are either measured or input values of the respective components.

After incorporating the transformation due to isolation transformer with ratio $n$ and coupling factor between primary and secondary turns of the isolation transformer is $k$, the net resistance and inductance in series equivalent matching circuit is given as,

$$
\begin{gathered}
R_{\text {final }}=\left(k^{2} n^{2} R_{p}\right), \\
L_{\text {final }}=\left(k^{2} n^{2} L_{\text {coil }}\right)+\left(1-k^{2}\right) L_{t}-\left(\frac{k^{2} n^{2}}{\omega^{2} C_{2}}\right),
\end{gathered}
$$

where $L_{t}$ is the inductance of isolation transformer and $C_{2}$ is the series capacitance in circuit.

Let net admittance $Y$ of the matching circuit (i.e. load to T-line) can be represented in the form of $Y=C+i D$. Considering the fig.3, the net admittance also can be written as,

$$
Y=\left(\frac{1}{z_{1}}+\frac{1}{z_{2}}\right)
$$

Total admittance of the equivalent circuit (fig.3) is the sum of admittance of shunt capacitor path and the path where series capacitor, coil inductor and the plasma load are placed in series. The arm of isolation transformer $\left(k^{2} L_{t}\right.$ term) is neglected because of low admittance and high inductance. The admittance by shunt capacitor $C_{1}$ is given as

$$
\frac{1}{z_{1}}=i \omega C_{1} .
$$

Net admittance of the second arm of the equivalent circuit (fig.2) is as,

$$
\frac{1}{Z_{2}}=\left\{\frac{R_{\text {final }}}{\left(R_{\text {final }}\right)^{2}+\left(\omega L_{\text {final }}\right)^{2}}\right\}-i\left\{\frac{\omega L_{\text {final }}}{\left(R_{\text {final }}\right)^{2}+\left(\omega L_{\text {final }}\right)^{2}}\right\},
$$

where

$$
Z_{2}=R_{\text {final }}+i \omega L_{\text {final }} .
$$

The load impedance $Z_{\text {load }}$ can be written in terms of admittance as,

$$
Z_{\text {load }}=\frac{1}{Y}=\left(\frac{C}{C^{2}+D^{2}}\right)+i\left(\frac{-D}{C^{2}+D^{2}}\right)=a+i b .
$$

$Z_{\text {load }}$ is the net load at transmission line. Net impedance seen by RF generator can be calculated from a standard relation [17].

$$
Z_{g}=Z_{0}\left[\frac{Z_{\text {load }}+i Z_{0} \tan \left(\frac{\omega l_{\text {lline }}}{c}\right)}{Z_{0}+i Z_{\text {load }} \tan \left(\frac{\omega l_{\text {lline }}}{c}\right)}\right]=P+i Q .
$$


Where $Z_{0}$ is the characteristic impedance of the line and $l_{\text {tline }}$ is the length of transmission line, $c$ is the speed of light with $\omega$ is RF frequency. In ideal condition $P$, the real part of impedance seen by RFG matching parameter should be raised to make $\mathrm{P} \sim \mathrm{Z}_{0}=50 \Omega$, $\mathrm{Q} \sim 0$ and $\operatorname{Cos} \phi$ must be as close to unity.

$$
\begin{array}{r}
P=\operatorname{Re}\left(Z_{g}\right), \\
Q=\operatorname{Im}\left(Z_{g}\right), \\
\cos \phi=\frac{P}{\sqrt{P^{2}+Q^{2}}} .
\end{array}
$$

For this model for RF impedance matching, it is clear that any change in $R_{p}$ (plasma resistance) due to time varying plasma condition (e.g. pressure variation) can affect the coupling and be visible in changing value of $\cos \phi$. The value can be restored back by tuning $C_{1}$ (coarse), $C_{2}$ (fine) and $\omega$ (fine). During impedance mismatched condition the amount of reflected power can be estimated as,

$$
P_{\text {reflected }}=P_{\text {forward }}\left[\frac{Z_{\text {load }}-Z_{0}}{Z_{0}+Z_{\text {load }}}\right]^{2} .
$$

The complete flowchart of this alternate method of impedance match tuning procedure is depicted in figure 4.

For the impedance matching the model requires a constant frequency $\omega$ which is not the case with an RFG of self-excited oscillator configuration. Any change in $R_{p}$ (plasma resistance) due to time varying plasma condition, can affect the stability of the frequency. The frequency stability is determined by the tank circuit and the working point of the oscillator tube. To incorporate this point a block is inserted in fig. -4 just at the junction of "model-a" and "model-b" to estimate $\omega$ of the RFG based on $R_{p}$ at that instant. The driver frequency $\omega$ is the input in "model-b".

The online impedance tuning of the matching circuit can be carried out by adjusting the variable capacitors $C_{1}, C_{2}$ through a motor driven mechanism. In a similar fashion, the online frequency $\omega$ tuning can be performed by adjusting the motor-driven tank circuit variable capacitor, placed inside the RF Generator. All these motor-driven capacitors, either in matching circuits or in tank circuit can be controlled by feedback algorithm in the control software using the data acquisition and control system of the experimental facility [15]. The capacitance value of these variable capacitors is calibrated with number of turns of the rotating shaft fitted with it. A set of metallic plates are attached with the rotating shaft and placed inside the gaps of another set of stationary metallic plates. By rotation, capacitance values are changed by changing the overlapping area between two sets of plates. 


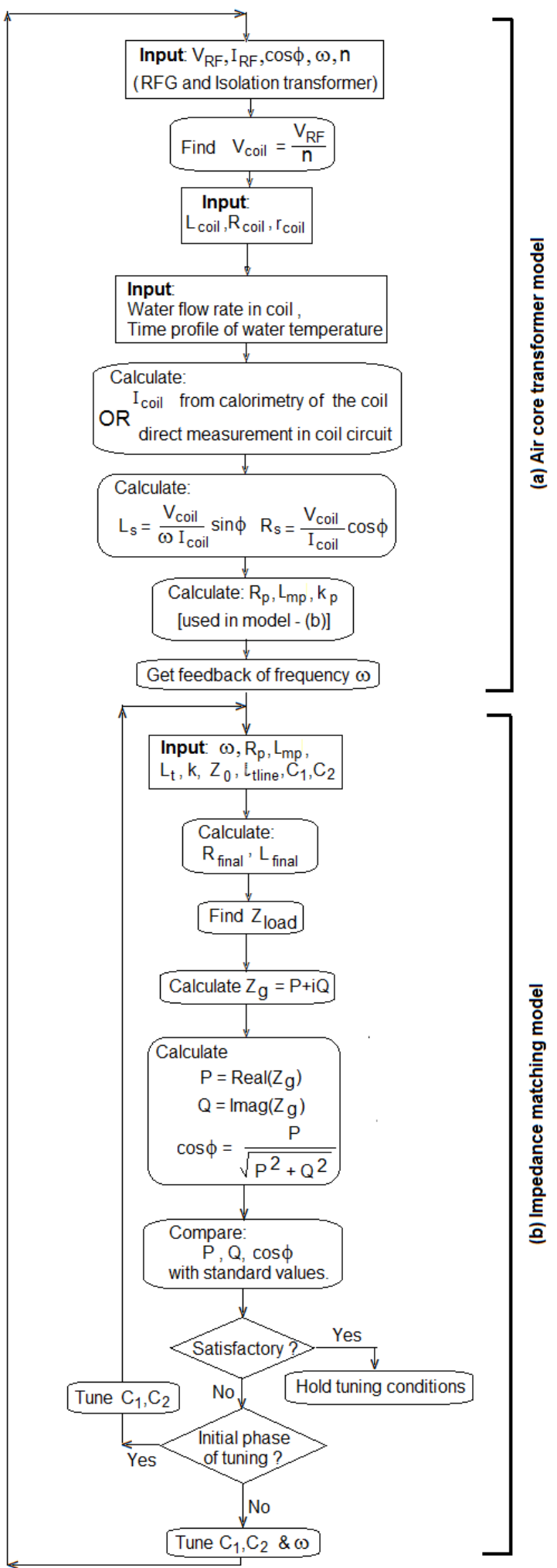

Fig. 4. Flowchart of online impedance matching algorithm taken into account of variation of plasma behavior. Initial phase indicates source "commissioning phase". 


\section{Results and discussion:}

Before using the models to study the effect on matching conditions due to change in plasma parameters, the above described models are validated with experimentally observed data using the experimental setup parameters in ROBIN facility [15]. These are,

Table -1 . Inputs from experimental condition, used for the model calculations

\begin{tabular}{|c|c|c|c|c|c|c|c|c|c|c|c|c|c|c|c|}
\hline \multicolumn{1}{|l|}{ RF generator initial inputs } & \multicolumn{1}{|c|}{ Matching circuit \& Transmission line } & \multicolumn{5}{c|}{ RF driver \& plasma } \\
\hline$\omega$ & $V_{R F}$ & $I_{R F}$ & $\cos \phi$ & $n$ & $k$ & $L_{t}$ & $l_{\text {tline }}$ & $C_{1}$ & $C_{2}$ & $Z_{0}$ & $L_{\text {coil }}$ & $R_{\text {coil }}$ & $r_{\text {coil }}$ & $P$ & Cal \\
\hline 1 & 2.65 & 25 & 0.75 & 3 & 0.95 & 70 & 5 & 3.0 & 2.65 & 50 & 12 & 0.733 & 0.15 & 0.3 & 3.5 \\
$M H z$ & $k V$ & $A$ & & & & $\mu H$ & $m$ & $n F$ & $n F$ & $\Omega$ & $\mu H$ & $\Omega$ & $m$ & $P a$ & $k W$ \\
\hline
\end{tabular}

In the above table-1, the values $k, L_{t}, L_{\text {coil }}$ are frequency dependent. However, for the present calculation values are taken as constant. Table- 1 indicates the list of input parameters (either measured or its intrinsic values), used to estimate the plasma resistance $R_{p}$ and plasma inductance $L_{m p}$. In the algorithm $R_{p}, L_{m p}$ along with above inputs are used in online impedance tuning purpose.

The frequency $\omega$ can be tuned by changing the capacitance value of the RFG tank circuit. In ROBIN, the RFG is tetrode based oscillator of maximum output power is $100 \mathrm{~kW}$. The tank circuit capacitance value can be change in discrete step by removing or adding capacitors in the tank circuit. In the present experiment the frequency is kept $\sim 1 \mathrm{MHz}$ (precisely $980 \mathrm{kHz}$ ). In upgraded version of the RFG for future experiments a tunable capacitor is envisaged for fine online frequency tuning.

$V_{R F}, I_{R F}$ and $\cos \phi$ are set based on RFG output power setting. Turn ratio $n$ in ferrite core isolation transformer placed in the matching circuit is 3. Coupling factor $k$ in between primary and secondary coil of the isolation transformer is 0.95 [18] and corresponding inductance is $70 \mu \mathrm{H}$. The length of $50 \Omega\left(\mathrm{Z}_{0}\right) \mathrm{RF}$ transmission line is $\sim 5 \mathrm{~m}$. All inputs for isolation transformer and transmission line are kept fixed for all the experimental study in the setup.

In the matching circuit, $C_{1}$ and $C_{2}$ are variable and adjusted to match the impedance between the RFG and the RF driver. In the initial phase of the experiment, $C_{1}, C_{2}$ are adjusted using equations ( model - b). The values of $\mathrm{C}_{1}$ and $\mathrm{C}_{2}$ are chosen as $3 n F$ and $2.65 n F$ respectively to get $P=\operatorname{Re}\left(Z_{g}\right) \sim 50 \Omega, Q=\operatorname{Re}\left(Z_{g}\right) \sim 0$ and $\cos \phi$ close to 1 . The value of $\mathrm{C}_{1}$ has low influence on matching condition, whereas the effect of $\mathrm{C}_{2}$ value is significant, as shown in fig.5. Fig.6 shows the possibility of impedance matching by frequency tuning. In this calculation plasma resistance $R_{p}$ involved in $R_{\text {final }}$ (finally in $Z_{g}$ ) is considered as $2.3 \Omega$ [18]. During experiment due to change in pressure and power, plasma parameters may change which affect the $R_{p}$. Equation -11 needs $I_{\text {coil }}$ apart for the inputs mentioned in table-1. $I_{\text {coil }}$ is estimated through coil calorimetric data assuming Joules heating by this current. Measurement accuracy of calorimetric data affects estimation of $R_{p}$. 
From RF coil cooling circuit 3.5kW power is removed during a plasma shot of using 50kW RF power. From this calorimetric data, $I_{\text {coil }}$ is estimated as $\sim 68 \mathrm{~A}$. Corresponding $V_{\text {coil }}$ is estimated from $V_{R F} / n$ as $880 \mathrm{~V}$. The ratio $V_{\text {coil }} / I_{\text {coil }}$ is $\sim 13$. In fig.7, the variation of plasma resistance $R_{p}$ and inductance $L_{m p}$ are calculated using equations -11 and 10 respectively. The value $R_{p}$ for $V_{\text {coil }} / I_{\text {coil }}$ $\approx 13$ is used to calculate $R_{\text {final }}$ for $P, Q, \cos \phi$ estimation.

The fig.7 and fig.8 give ideas about the range of $R_{p}$ during a plasma shot due to plasma parameter and pressure variations. Fig. 9 gives the corresponding range of online tuning. This figure indicates that a small variation in $R_{p}$ (due to variation of discharge conditions) can lead to detuning of the matching circuit. In that case further tuning can be carried out by $C_{1}, C_{2}$ and $\omega$ for a particular isolation transformer.
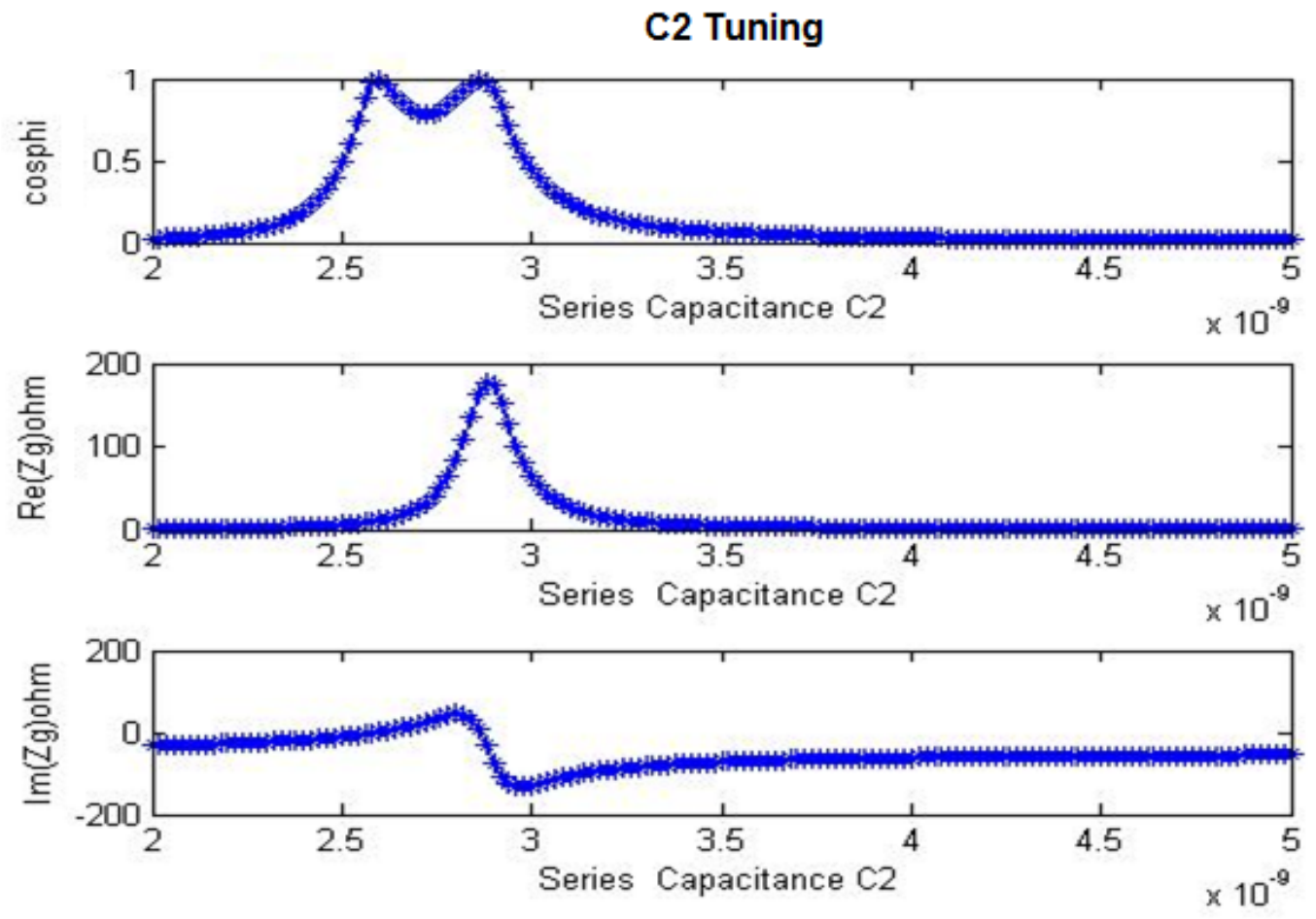

Fig.5. Identifying the values of series capacitor $C_{2}$, for tuning the impedance matching circuit $[\operatorname{Re}(\mathrm{Zg}) \rightarrow 50 \Omega, \cos \phi \rightarrow 1$ and $\operatorname{Im}(\mathrm{Zg}) \rightarrow 0]$ during initial phase of experiment, using a computer code developed by $W$. Kraus. The value of shunt capacitor is considered as $3 \mathrm{nF}$. 

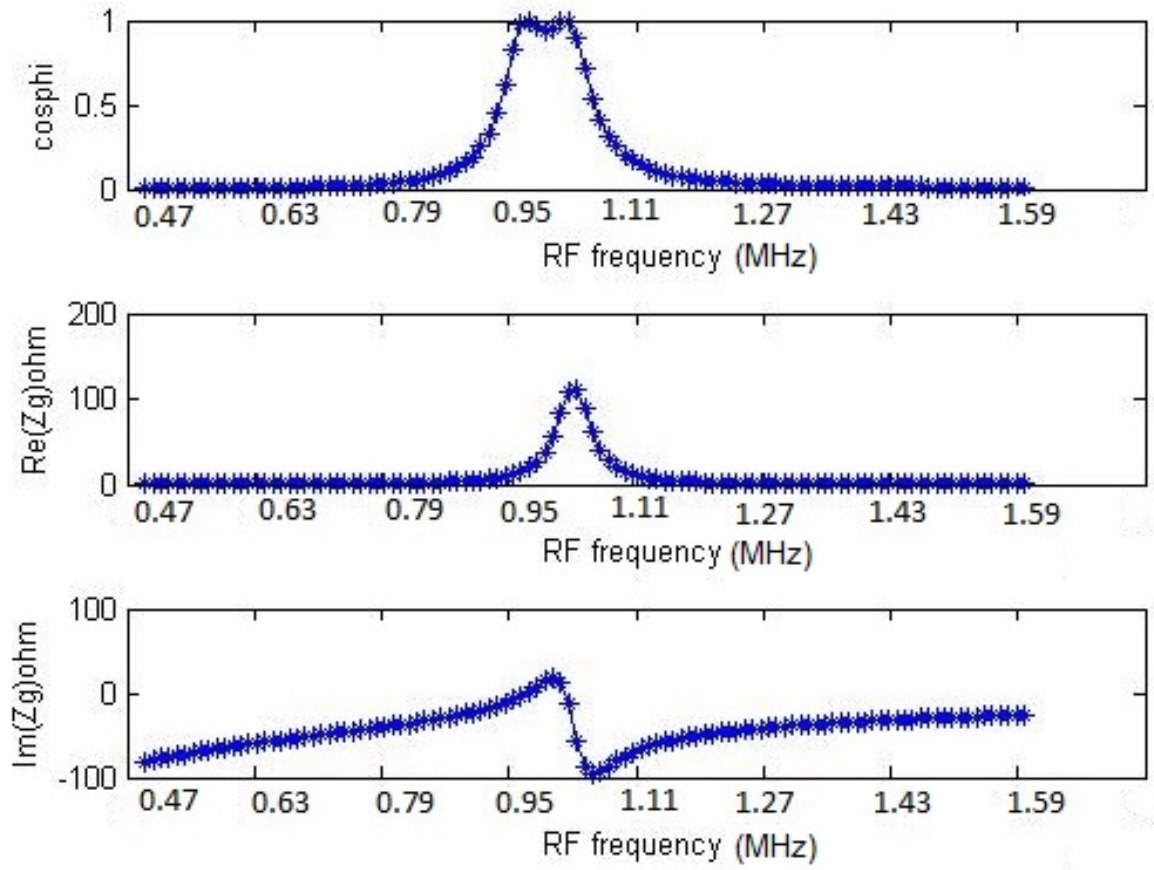

Fig.6. Possibility of impedance matching by frequency tuning.
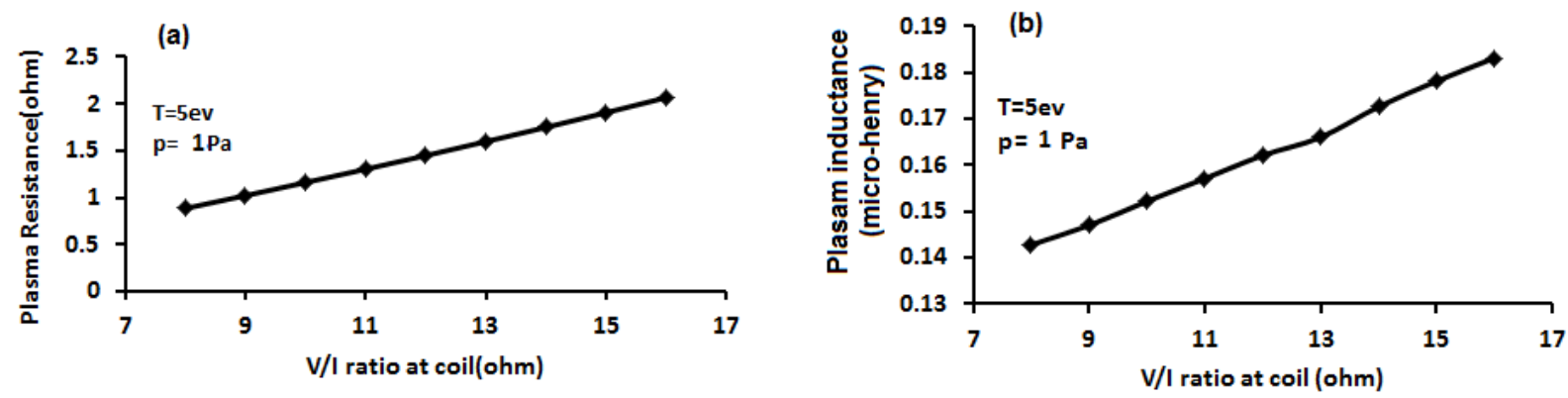

Fig.7. (a) Variations of estimated plasma resistance $R_{p}$ for different V/I conditions corresponding to $1 \mathrm{~Pa}$ pressure and $5 \mathrm{eV}$ plasma temperature. $R_{p}$ values from the Transformer model are estimated considering $C_{1}=3 \mu \mathrm{F}$ and $C_{2}=2.65 \mu \mathrm{F}$. Experimental condition satisfies $V / I \approx 13$. (b) Variations of plasma inductance $L_{m p}$ for different $V / I$ values corresponding to the same plasma conditions. 

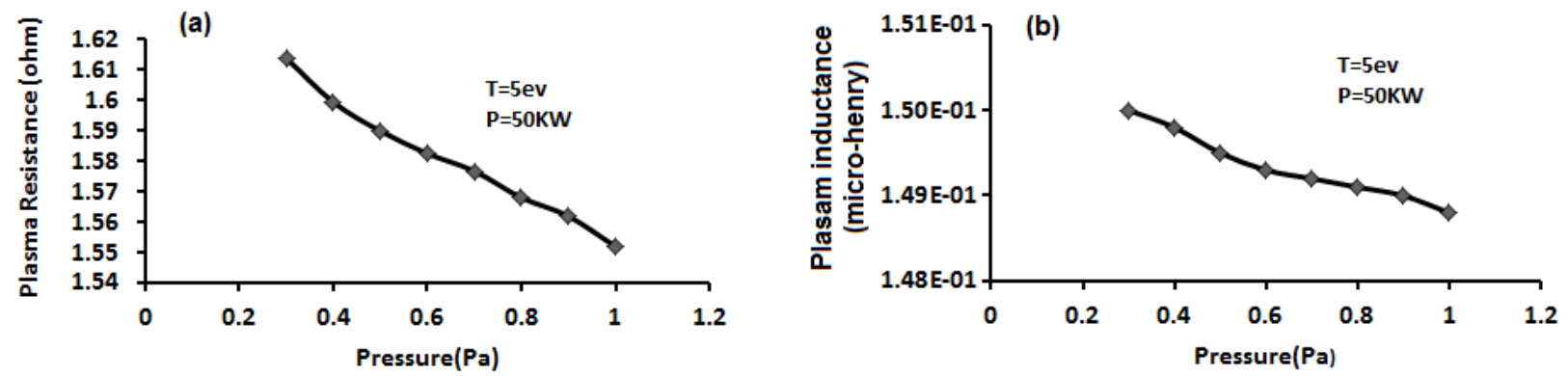

Fig.8. (a) Variations of estimated plasma resistance $R_{p}$ for different values of operating pressure corresponding to $50 \mathrm{~kW}$ of RF power and $5 \mathrm{eV}$ plasma temperature. (b) Variations of plasma inductance $L_{m p}$ for different values of operating pressure corresponding to the same plasma conditions.

(a)

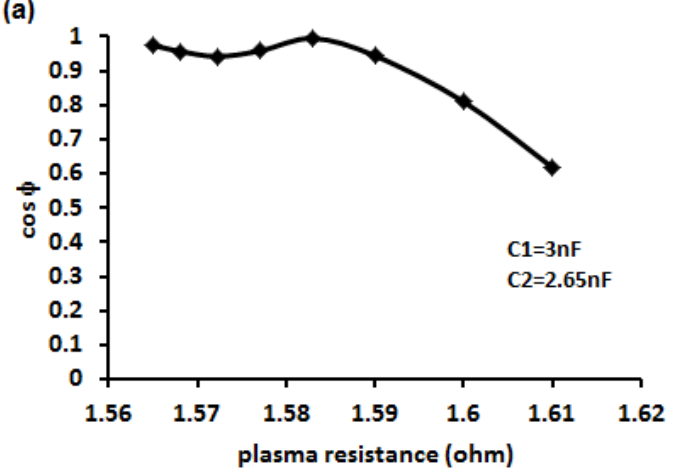

(b)

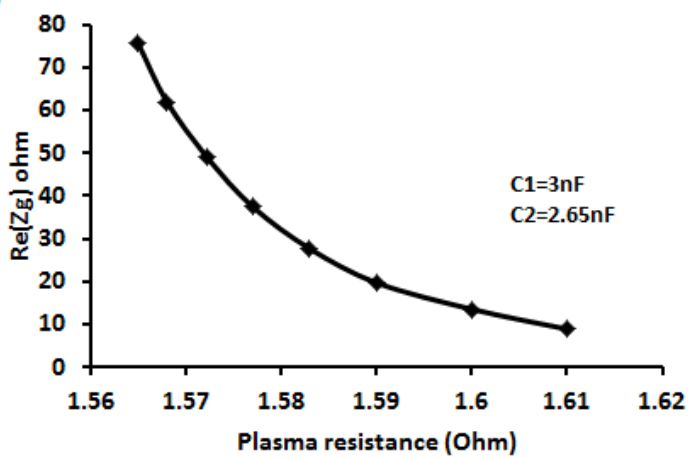

Fig.9. Range of tuning (a) $\cos \phi$ and (b) Real part of $\mathrm{Zg}$ in terms of $R_{p}$ variation. Small variation in $R_{p}$ leads to large variation in tuning parameters. Plasma inductance is negligibly small (see fig. 5,6).
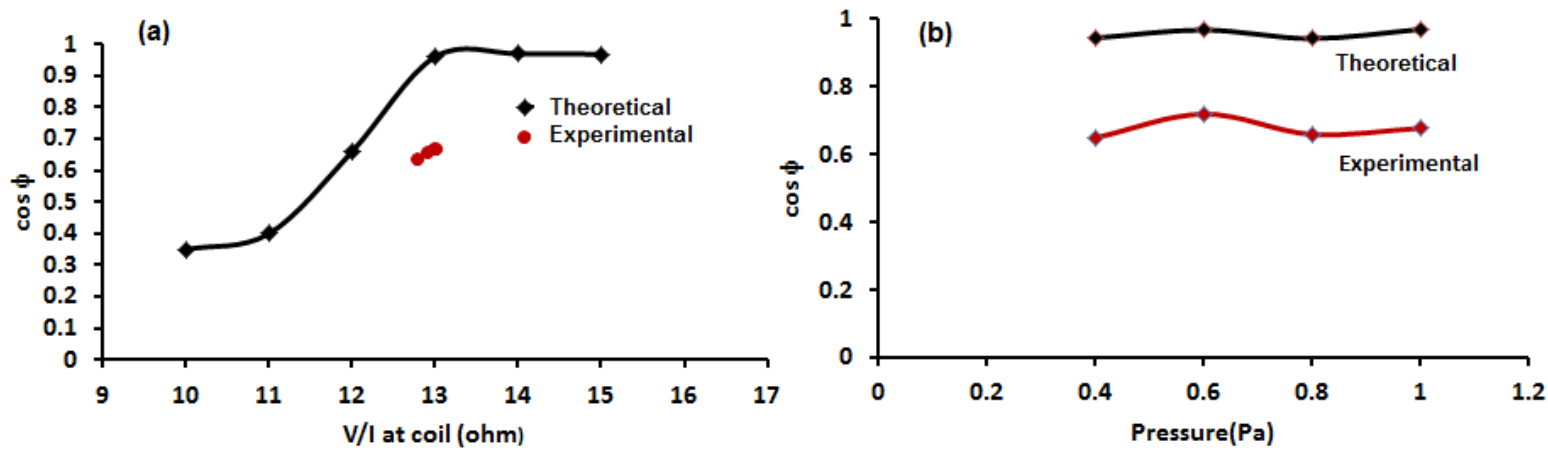

Fig.10. Comparison of $\cos \phi$ values (matching condition) from model calculation and corresponding experimental data. In (a) experimental data are corresponding to 30, 40, $50 \& 60 \mathrm{~kW}$ inductive discharge power and in (b) experimental data are corresponding to different operational gas pressure. 
Experimental matching condition $\cos \phi$ is compared with the theoretical calculation based on the model described in the paper and shown in fig.10. The present transformer model is purely treating the plasma as an electrical component. It is seen from fig.9 that a small variation in $R_{p}$ may change the matching condition significantly. Fig. $9 \mathrm{~b}$ is showing the real part of $Z_{g}$ variation with plasma resistance $R_{p}$. However, matching condition is not varied much, which can be understood by $\cos \phi$ plot in fig.9a. The value $\cos \phi$ is the only measurable parameter in the experimental system to indicate the matching condition. If in fig. $9 \mathrm{~b}$ the $\operatorname{Real}\left(Z_{g}\right)$ is changed from $75 \mathrm{Ohm}$ to $10 \mathrm{Ohm}$, when the plasma resistance is changed only by $0.04 \mathrm{Ohm}$, the RF power would be reflected significantly. May be this is correct and it is caused by the slope in fig5 [Real $\left(Z_{g}\right)$ vs C2], but in reality it is stabilized by a simultaneous change of the RF generator frequency (slope in Fig6 Real $\left(Z_{g}\right) v s$ Frequency). So in reality, during the source operation the variation of $\operatorname{Real}(\mathrm{Zg})$ is much less pronounced. To accommodate this frequency change due to real(Zg), a "frequency block" is incorporated into the flowchart (fig. - 4 ). In particular, it could explain the matching to a self-excited oscillator.

The value of $R_{p}$ depends on the parameters: like; coupling coefficient $k$ of the isolation transformer, the accuracy of calorimetric data and the plasma dynamics. In the present case, $k=0.95$ is assumed considering excellent magnetic linkage between the primary and the secondary of the isolation transformer, which may be in higher side and optimistic.

For calorimetric measurement the output temperature is measured by a thermocouple placed in the return path, 3m away from the RF coil terminal (away from RF environment) and the water flow is measured by a rotameter in the RF coil cooling circuit. Calorimetric measurement is a slow process which may influence the timescale of the proposed tuning mechanism.

The tuning mechanism discussed so far, has considered the presence of plasma after the ignition phase. During plasma ignition in such sources, rapid change in plasma impedance takes place in the time scale of milisecond or even less. The matching algorithm based on calorimetric measurement of coil current is not compatible with this time scale. However, remaining part of the algorithm is still useful to handle such rapid phenomena. There is another limitation of matching during ignition arising from hardware. The DAC - motor system may not be fast enough to respond in that time scale.

In the experiment with hydrogen gas in ROBIN, ignition phase initiated with a filament heating and corresponding arc discharge of $\sim$ few $\mathrm{mA}$ discharge current, which provide the presence of seed electrons (pre-ionization) to gain energy from the RF field and ignite the plasma inside the $\mathrm{RF}$ coil. To ensure ignition, a gas puff is also applied $\sim 1 \mathrm{~Pa}$ or more for $\sim 200 \mathrm{~ms}$, just at the strike point of RF generator power supply. Once the ignition is successful, pressure is reduced to normal operation mode, of the range $\sim 0.3-1.0 \mathrm{~Pa}$ within $\sim 1 \mathrm{~s}$. Plasma ignition is monitored through observing $\mathrm{H}$-alpha radiation (656.3 $\mathrm{nm}$ wavelength) from the plasma. The $\mathrm{H}$-alpha signal level is also used as an interlock with the RF generator to switch it off in the case RF ignition does not strike properly. This interlock is to protect the RF generator from damage due to the 
reflected power, $\sim$ few tens of kilo watt; incase plasma is not formed (unsuccessful ignition i.e. unmatched load condition). A description of the source operation can be found in the reference 15.

The physics understanding on ICP discharge is not folded in the present model. The expressions for $k_{p}$ in equation (9) are considered from Peizek model [14] which may be in-adequate to consider all the physics involved for high power $\sim 50 \mathrm{~kW}$ ICP discharge. In this power level nonlinear, non-local, collision less phenomena for plasma formation and its dynamics are dominant [19, 20, 21, 22]. Apart from that antenna configuration including geometrical factors plays a role as well in coupling factor [11]. A separate study on these effects is being carried out and will be reported elsewhere.

\section{Summary:}

The present manuscript tries to combined transformer model for ICP discharge with the impedance matching calculation to develop an online impedance matching methodology. This technique may be useful for long pulse ICP discharge. Matching condition influences power absorption into the plasma through the RF coil antenna. Online time dependent calorimetric data from the coil cooling circuit has the information of time dependent power delivery to the plasma. This information is used in the present model for online tuning mechanism. Caloimetric measurement is a slow process which may influence the promptness of the proposed tuning mechanism. The discrepancies observed between the theoretical calculations using the present model and the experimental data are attributed to the accurate value of isolation transformer coupling coefficient, accuracy of calorimetric data and the physics limitations involved in the model which are under investigation. This paper is trying to establish an alternate remote online tuning methodology for long pulse operation without arguing its superiority over other techniques.

\section{Acknowledgement}

Authors are grateful to IPP's NNBI team and IPR's NIST group for their constant technical help during ROBIN commissioning and operation. Discussion with them during this period is the motivation of this work. D. Sudhir would like to thank Mr. Himanshu Tyagi for his help to write a program based on the present model for calculation and corresponding plotting.

\section{References:}

[1] W. Hittorf, Ann. Phys., Lpz., 21, 137, (1884).

[2] T. H. Stix and R. W. Palladino, Phys. Fluids, 1, 446, (1958).

[3] J. Hopwood, Plasma Sources Sci. Technol., 1, 109, (1992).

[4] E. F. Jaeger, L. A. Berry, J. S. Tolliver, and D. B. Batchelor, Phys. Plasmas 2, 2597 (1995). 
[5] E. Speth, H.D.Falter, P. Franzen, U Fantz, M. Bandyopadhyay, S. Christ, A. Encheva, M. Froschle, D. Holtum, B. Heinamann, W. Kraus, A. Lorenz, Ch. Marten, P. McNeely, S. Obermayer, R. Riedl, R. Suss, A. Tanga, R. Wilhelm and D. Wunderlich, Nucl. Fusion, 46, S220, (2006).

[6] R. Hemsworth, H. Decamps, J. Graceffa, B. Schunke, M. Tanaka, M. Dremel, A. Tanga, H.P.L.De. Esch, F. Geli, J. Milnes, T. Inoue, D. Marcuzi, P. Sonato and P. Zaccaria, Nucl. Fusion, 49, 045006, (2009).

[7] P. Sonato, P. Agostinetti, G. Anaclerio, V. Antoni, O. Barana, M. Bigi, M. Boldrin, M. Cavenago, D. Dal Bello, M. Dalla Palma et al., Fusion Engineering and Design, 84, 269, (2009).

[8] M.J. Singh, M.Bandyopadhyay, C. Rotti, N.P.Singh, S. Shah, G. Bansal, A. Gahlaut, J. Soni, H. Lakdawala, H. Waghela, I. Ahmed, G. Roopesh, U.K.Baruah and A.K.Chakraborty, 86, 732, Fusion Engineering \& Design (2011).

[9] D. Marcuzzi, M. Dalla Palma, M. Pavei, B.Heinamann, W. Kraus and R. Riedl, Fusion Engineering and Design 84, 1253, (2009).

[10] A.Chakraborty, C.Rotti, M. Bandyopadhyay, M. J. Singh, Roopesh G, Sejal Shah, U.K.Baruah, R. Hemsworth and B. Schunke, IEEE Transactions on Plasma Science, 38, 248, (2010).

[11] Yaoxi Wu and M. A. Libermann, Plasma, Sources Sci. Technol., 9, 210, (2000).

[12] J. W. Denneman, J . Phys. D: Appl. Phys. 23 293, (1990).

[13] G. G. Lister and M. Cox, Plasma, Sources Sci. Technol., 1, 67, (1992).

[14] R. B. Piejak, V. A. Godyak and B. M. Alexandrovich, Plasma Sources Sci. Technol., 1,179, (1992).

[15] M.J. Singh, M. Bandyopadhyay, G. Bansal, A. Gehlaut, J. Soni, S. Kumar, K. pandya, K.G.Parmer, J. Snara, R. Yadav, A.K.Chakraborty, W. Kraus,B. Heinamenn, R. Riedl, S. Obermayer, C. Martens, P. Franzen and U. Fantz., Proc. $2^{\text {nd }}$ Int. Symp. Neg. Ions, Beams and Sources, AIP conf. 1390, 604, (2011).

[16] G. Bansal, A. Gahlaut, J. Soni, K. Pandya, K.G. Parmar, R. Pandey, M. Vuppugalla, B. Prajapati, A. Patel, H.Mistery, A.K.Chakraborty, M. Bandyopadhyay, M.J. Singh, A. Phukan, R. K. Yadav and D. Parmar, Proc. Symp. Fusion Technology (SOFT), (2012).

[17] Specification document of 100kW RFG model HGL 100-l, Himmelwerk, Tubingen, Germany.

[18] P. Chabert and N. Braithwaite, Physics of Radio-frequency Plasmas, Cambridge Univ. Press., UK, ISBN 978-0-521-76300-4, (2011).

[19] W. Kraus, Private communication.

[20] V. A. Godyak, Phys. Plasma, 12, 055501, (2005).

[21] F. A. Haas, Plasma, Sources Sci. Technol., 10, 440, (2001).

[22] G. J. M. Hagelaar, Phys. Rev.Lett. 100, 025001, (2008).

[23] R. Wilhelm, Phys. Plasma, 10, 513, (2003). 